\title{
DECOMPOSABILITY OF HOMOTOPY LENS SPACES
}

\author{
BY KAI WANG \\ Communicated by Glen E. Bredon, September 25, 1972
}

Let $A$ be the antipodal map of $S^{p}$ and $D^{p+1}$. Let $f$ be an equivariant diffeomorphism of $\left(S^{p} \times S^{p}, A \times A\right)$. Then there is a well-defined free involution $A(f)$ on $\Sigma(f)$, where

$$
\Sigma(f)=S^{p} \times D^{p+1} \cup_{f} D^{p+1} \times S^{p}
$$

such that $A(f) \mid S^{p} \times D^{p+1}=A \times A$ and $A(f) \mid D^{p+1} \times S^{p}=A \times A$. In [2] G. R. Livesay and C. B. Thomas have shown that any free involution $\left(\Sigma^{2 p+1}, T\right)$ on the homotopy sphere $\Sigma^{2 p+1}$ is decomposable, i.e., there is an equivariant diffeomorphism $f$ of $\left(S^{p} \times S^{p}, A \times A\right)$ such that $\left(\Sigma^{2 p+1}, T\right)$ is equivalent to $(\Sigma(f), A(f))$. For $p=$ odd, let $A$ be a linear $Z_{n}$ action on $S^{p}$ and $D^{p+1}$. We can generalize the notion of decomposable actions to $Z_{n}$ actions. Using the same argument, they have shown that all free $Z_{3}$ actions on homotopy spheres are decomposable. The proof uses the following two well-known facts: (a) $J: K O\left(R P^{p}\right) \rightarrow J\left(R P^{p}\right)$ and

$$
J: K O\left(L^{4 n-1}\left(Z_{3}\right)\right) \rightarrow J\left(L^{4 n-1}\left(Z_{3}\right)\right)
$$

are isomorphisms and (b) $\mathrm{Wh}\left(Z_{2}\right)$ and $\mathrm{Wh}\left(Z_{3}\right)$ are zero. The argument breaks down for $Z_{n}$ actions, for $n \geqq 4$. Hence they asked if there are similar properties for $Z_{n}$ actions, for $n \geqq 4$ ([2], [3]). On the other hand, we have studied the analogs for free actions of $S^{1}$ and $S^{3}$ on homotopy spheres. The same argument works if we replace (a) by some restrictions on the orbit spaces [7].

In this paper we will show that certain free $Z_{n}$ actions on homotopy spheres are decomposable and the restrictions are nontrivial and necessary.

For $\varepsilon=h$ or $s$, let $\mathscr{S}^{\varepsilon}\left(L^{2 m 1}\right)$ be the set of (simple, if $\varepsilon=s$ ) homotopy of complex dimension $m$. Then $\rho=\sum_{j=1}^{m} t^{r_{j}}$ where $t$ is the basic complex one dimensional representation of $Z_{n}$ defined to be the multiplication by $\exp (2 \pi i / n)$. Let $p=[m / 2], q=m-[m / 2]$. It is clear that

$$
S^{2 m-1}, S^{2 p-1} \times D^{2 q}, D^{2 p} \times S^{2 q-1} \text { and } S^{2 p-1} \times S^{2 q-1}
$$

are invariant subspaces. Let

AMS(MOS) subject classifications (1970). Primary 57E30.

Key words and phrases. Free $Z_{n}$ actions, Wall groups, simple homotopy smoothings, decomposable actions, homotopy lens space. 


$$
\begin{aligned}
L^{2 m-1} & =L^{2 m-1}\left(n ; r_{1}, \ldots, r_{m}\right)=S^{2 m-1} / \rho, \\
M^{2 m-1} & =M^{2 m-1}\left(n ; r_{1}, \ldots, r_{m}\right)=S^{2 p-1} \times D^{2 q} / \rho, \\
N^{2 m-1} & =N^{2 m-1}\left(n ; r_{1}, \ldots, r_{m}\right)=D^{2 p} \times S^{2 q-1} / \rho, \\
K^{2 m-2} & =K^{2 m-2}\left(n ; r_{1}, \ldots, r_{m}\right)=S^{2 p-1} \times S^{2 q-1} / \rho .
\end{aligned}
$$

For $\varepsilon=h$ or $s$, let $\mathscr{S}^{\varepsilon}\left(L^{2 m-1}\right)$ be the set of (simple, if $\varepsilon=s$ ) homotopy smoothings of $L^{2 m-1}$ and $G / O$ be the classifying space for $G / O$-bundles and let $L_{2 m}\left(Z_{n}\right)^{0}$ be the reduced Wall group of $Z_{n}$. By the theorem of T. Petrie [5] (see also [1]) $L_{2 m}\left(Z_{n}\right)^{0}$ acts freely on $\mathscr{S}^{s}\left(L^{2 m-1}\right)$. Let

$$
\eta: \mathscr{S}^{\varepsilon}\left(L^{2 m-1}\right) \rightarrow\left[L^{2 m-1}, G / O\right]
$$

be the normal map. W. Browder [1] has proved that $\eta$ is onto if $n$ is odd and according to C. T. C. Wall [6] the coker $\eta$ is $Z_{2}$ if $n$ is even. Let

Let $L^{2 p-1}=L^{2 p-1}\left(n ; r_{1}, \ldots, r_{p}\right)$ and $L^{2 q-1}=L^{2 q-1}\left(n ; r_{p+1}, \ldots, r_{m}\right)$.

$$
\begin{aligned}
& t_{1}^{\varepsilon}: \mathscr{S}^{\varepsilon}\left(L^{2 m-1}\right) \stackrel{\eta}{\rightarrow}\left[L^{2 m-1}, G / O\right] \rightarrow\left[L^{2 p-1}, G / O\right], \\
& t_{2}^{\varepsilon}: \mathscr{S}^{\varepsilon}\left(L^{2 m-1}\right) \stackrel{\eta}{\rightarrow}\left[L^{2 m-1}, G / O\right] \rightarrow\left[L^{2 q-1}, G / O\right], \\
& \bar{t}_{1}^{\varepsilon}: \mathscr{S}^{\varepsilon}\left(L^{2 m-1}\right) \stackrel{t_{1}^{\varepsilon}}{\rightarrow}\left[L^{2 p-1}, G / O\right] \rightarrow\left[L^{2 p-1}, B O\right], \\
& \bar{t}_{2}^{\varepsilon}: \mathscr{S}^{\varepsilon}\left(L^{2 m-1}\right) \stackrel{t_{2}^{\varepsilon}}{\longrightarrow}\left[L^{2 q-1}, G / O\right] \rightarrow\left[L^{2 q-1}, B O\right] .
\end{aligned}
$$

Proposition 1. Let $X^{2 m-1}$ be a closed manifold which is homotopy equivalent to $L^{2 m-1}\left(n ; r_{1}, \ldots, r_{m}\right)$. Suppose that there is an $h$-cobordism $W^{2 m-1}$ of $K^{2 m-2}\left(n ; r_{1}, \ldots, r_{m}\right)$ to itself so that

$$
X^{2 m-1} \cong M^{2 m-1}\left(n ; r_{1}, \ldots, r_{m}\right) \cup W^{2 m-1} \cup N^{2 m-1}\left(n ; r_{1}, \ldots, r_{m}\right) .
$$

Then for any homotopy equivalence

$$
f: X^{2 m-1} \rightarrow L^{2 m-1}\left(n ; r_{1}, \ldots, r_{m}\right),
$$

$t_{i}^{h}\left(\left[X^{2 m-1}, f\right]\right)=0$ and $t_{i}^{s}\left(\left[X^{2 m-1}, f\right]\right)=0$ if $f$ is a simple homotopy equivalence, $i=1,2$.

It is easy to see that for $n \geqq 4$, there is $x \in\left[L^{2 m-1}, G / O\right]$ such that $x \mid L^{2 p-1} \neq 0$ and if $n$ is even we may choose $x \in \operatorname{Im} \eta$. Suppose $x=$ $\eta\left(\left[X^{2 m-1}, f\right]\right)$. Hence $X^{2 m-1}$ cannot be decomposable. Furthermore, for $w \in L_{2 m}\left(Z_{n}\right)^{0}$,

$$
t_{1}^{s}\left(w+\left[X^{2 m-1}, f\right]\right)=t_{1}^{s}\left(\left[X^{2 m-1}, f\right]\right) \neq 0 .
$$

Since rank $L_{2 m}\left(Z_{n}\right)^{0} \geqq 1$ for $n \geqq 4[5]$, we have

COROLlARY 2 . For $m \geqq 3$ and $n \geqq 4$, there are infinitely many inequiva- 
lent nondecomposable free $Z_{n}$ actions on homotopy $(2 m-1)$-spheres of which the orbit spaces are simple homotopy equivalent to $L^{2 m-1}\left(n ; r_{1}, \ldots, r_{m}\right)$.

THEOREM 3. Let $X^{2 m-1}$ be a closed manifold which is homotopy equivalent to $L^{2 m-1}\left(n ; r_{1}, \ldots, r_{m}\right)$. Suppose that there is a simple homotopy equivalence

$$
f: X^{2 m-1} \rightarrow L^{2 m-1}\left(n ; r_{1}, \ldots, r_{m}\right)
$$

such that $\bar{t}_{i}^{s}\left(\left[X^{2 m-1}, f\right]\right)=0, i=1,2$. Then $X^{2 m-1}$ is decomposable.

The proof can be sketched as follows. Let $f^{\prime}$ be the homotopy inverse of $f$. By assumption, we can modify $f^{\prime}$ to make $f^{\prime} \mid M^{2 m-1}$ and $f^{\prime} \mid N^{2 m-1}$ be embeddings. Then

$$
W=X^{2 m-1}-\operatorname{int} f^{\prime}\left(M^{2 m-1}\right)-\operatorname{int} f^{\prime}\left(N^{2 m-1}\right)
$$

is an $h$-cobordism of $K^{2 m-2}$ to itself. When $f$ is a simple homotopy equivalence, we can show that $W$ is equivalent to a product. Therefore, $X^{2 m-1}$ is decomposable.

Suppose there is an $h$-cobordism $W$ of $K^{2 m-2}$ to itself such that $X^{2 m-1}=M^{2 m-1} \cup W \cup N^{2 m-1}$. We can choose a homotopy equivalence $h: X^{2 m-1} \rightarrow L^{2 m-1}$ such that $h \mid W: W \rightarrow K^{2 m-2} \times[0,1]$ is a homotopy equivalence and $h \mid \partial W$ is a diffeomorphism. Then $\tau(h)=i_{*} \tau(h \mid W)$ where $i_{*}: \mathrm{Wh}\left(\pi\left(K^{2 m-2} \times[0,1]\right)\right) \rightarrow \mathrm{Wh}\left(\pi\left(L^{2 m-1}\right)\right)$ induced by

$$
i: K^{2 m-2} \times[0,1] \hookrightarrow L^{2 m-1}
$$

and $\tau(h)$ is the torsion of $h$. If $X^{2 m-1}$ is decomposable $\tau(h \mid W)=0$. Hence $\tau(h)=0$. Thus we have proved

THEOREM 4. Let $X^{2 m-1}$ be a closed manifold which is homotopy equivalent to $L^{2 m-1}\left(n ; r_{1}, \ldots, r_{m}\right)$. Suppose $X^{2 m-1}$ is decomposable, then $X^{2 m-1}$ is simple homotopy equivalent to $L^{2 m-1}\left(n ; r_{1}, \ldots, r_{m}\right)$.

For $u \in 2 \mathrm{Wh}\left(Z_{n}\right)$, there is an $h$-cobordism $W_{u}$ of $K^{2 m-2}$ to itself such that $\tau\left(W_{u}, K\right)=u$. Let $X_{u}=M^{2 m-1} \cup W_{u} \cup N^{2 m-1}$. Let $\widetilde{W}_{u}$ and $\widetilde{X}_{u}$ be the universal coverings of $W_{u}$ and $X_{u}$ respectively. $\tilde{W}_{u}$ is an $h$-cobordism of $S^{2 p-1} \times S^{2 q-1}$ to itself. $S^{2 p-1} \times S^{2 q-1}$ is simply-connected. Then $\tilde{W}_{u} \simeq S^{2 p-1} \times S^{2 q-1} \times[0,1]$ and $\tilde{X}_{u}$ is a homotopy sphere supporting a free $Z_{n}$ action with orbit space $X_{u}$. $W_{u}$ is homotopy equivalent to $K^{2 m-2} \times$ $[0,1]$. Let $H_{u}: W_{u} \rightarrow K^{2 m-2} \times[0,1]$ be a homotopy equivalence such that $H_{u} \mid \partial W_{u}$ is a diffeomorphism. Let $h_{u}=\mathrm{id} \cup H_{u} \cup$ id. Then $h_{u}: X_{u} \rightarrow$ $L^{2 m-1}$ is a homotopy equivalence and $\tau\left(h_{u}\right)=u$.

COROllaRY 5. For $n \geqq 4$, there are infinitely many closed manifolds $X_{u}^{2 m-1}$ which are homotopy equivalent to $L^{2 m-1}\left(n ; r_{1}, \ldots, r_{m}\right)$ such that $t_{i}^{h}\left(X_{u}^{2 m-1}\right)=0, i=1,2$, but none of $X_{u}^{2 m-1}$ are simple homotopy equivalent to $L^{2 m-1}\left(n ; r_{1}, \ldots, r_{m}\right)$. 
Using the same techniques in [7] we can prove

THEOREM 6. Let $\left(\Sigma^{2 m-1}, Z_{n}\right)$ be a decomposable free $Z_{n}$ action on homotopy sphere $\Sigma^{2 m-1}$. Then $\Sigma^{2 m-1}$ supports infinitely many inequivalent free $Z_{n}$ actions of which the orbit spaces are of same simple homotopy type.

REMARK 7. If $r_{i}=r_{p+i}, i=1, \ldots, p$. Then the condition in Theorem 3 can be weakened to require that $\bar{t}_{2}^{s}\left(\left[X^{2 m-1}, f\right]\right)=0$.

REMARK 8. Suppose $n$ is odd. Let $A: \mathscr{S}^{s}\left(L^{2 k-1}\right) \rightarrow C^{Z_{n}-1}$ be the AtiyahSinger invariants [5]. For $x \in\left[L^{2 k-1}, G / O\right]$, let $x=\eta(a)$ for $a \in \mathscr{S}^{s}\left(L^{2 k-1}\right)$. Define $\bar{A}(x)=A(a)$. Using results in [6] it is easy to show that $\bar{A}$ is well defined. Let

$$
\begin{aligned}
& T_{1}: \mathscr{S}^{s}\left(L^{2 m-1}\right) \stackrel{\eta}{\rightarrow}\left[L^{2 m-1}, G / O\right] \rightarrow\left[L^{2 p-1}, G / O\right] \stackrel{\bar{A}}{\rightarrow} C^{Z_{n}-1} \\
& T_{2}: \mathscr{S}^{s}\left(L^{2 m-1}\right) \stackrel{\eta}{\rightarrow}\left[L^{2 m-1}, G / O\right] \rightarrow\left[L^{2 q-1}, G / O\right] \stackrel{A}{\rightarrow} C^{Z_{n}-1} .
\end{aligned}
$$

Then we can replace the condition in Theorem 3 by requiring that $T_{i}\left(\left[X^{2 m-1}, f\right]\right)=0, i=1,2$.

REMARK 9. Theorem 3, Theorem 4 and Theorem 5 have been independently obtained by Chao-chu Liang, who is a student of G. R. Livesay.

\section{REFERENCES}

1. W. Browder, Free $Z_{p}$-actions on homotopy spheres, Proc. Georgia Conf. in Topology of Manifolds (Inst. Univ. of Georgia, Athens, Ga., 1969), Markham, Chicago, Ill., 1970, pp. 217-226. MR 43 \#2720.

2. G. R. Livesay and C. B. Thomas, Free $Z_{2}$ and $Z_{3}$ actions on homotopy spheres, Topology 7 (1968), 11-14. MR 36 \#3343.

3. G. R. Livesay and C. B. Thomas, Problem 9 in "Problems", Proc. Conf. on Transformation Groups (New Orleans, 1967), Springer-Verlag, Berlin and New York, 1968, pp. 235-236.

4. J. Milnor, Whitehead torsion, Bull. Amer. Math. Soc. 72 (1966), 358-426. MR 33 \#4922.

5. T. Petrie, The Atiyah-Singer invariant, the Wall groups and the function $\left(t e^{x}+1\right) /\left(t e^{x}-1\right)$, Ann. of Math. (2) 92 (1970), 174-187.

6. C. T. C. Wall, Surgery on compact manifolds, Springer-Verlag, Berlin and New York, 1970.

7. K. Wang, Free and semi-free smooth actions of $S^{1}$ and $S^{3}$ on homotopy spheres, Proc. Conf. on Transformation Groups (Amherst, 1971), Springer-Verlag, Berlin and New York, 1972.

Department of Mathematics, University of Chicago, Chicago, Illinois 60637

Institute for Advanced Study, Princeton, New Jersey 08540 (Current address.) 